\title{
Role of the Hippocampal-Entorhinal Loop in Temporal Lobe Epilepsy: Extra- and Intracellular Study in the Isolated Guinea Pig Brain in vitro
}

\author{
Denis Paré, ${ }^{1}$ Marco deCurtis, ${ }^{1,2}$ and Rodolfo Llinás' \\ 'Department of Physiology and Biophysics, New York University Medical Center, New York, New York 10016 and \\ 2Dipartimento di Neurofisiologia, Istituto Neurologico, 20133 Milano, Italy
}

\begin{abstract}
This article introduces a new experimental paradigm for the study of temporal lobe epilepsy. This approach utilizes the isolated guinea pig brain in vitro preparation, which generates a pattern of hypersynchronous neuronal activity similar to the peculiar 8-30 $\mathrm{Hz}$ rhythm characterizing stereoelectroencephalographic hippocampal recordings in human temporal lobe epilepsy. The present report describes an attempt to identify the functional events underlying the epileptiform activities observed in this preparation.
\end{abstract}

Rhythmic epileptiform discharges (EDs), here defined as population spikes (PSs) recorded from somata or dendritic layers, were Induced in the hippocampal formation of the isolated guinea pig brain maintained in vitro by tetanic stimulation of the entorhinal cortex (EC). Two patterns of EDs were distinguished by performing simultaneous field potential recordings along the dentate gyrus (DG), EC, CA1, and CA3. During stage 1, the first self-sustained EDs were isolated PSs occurring at a frequency of 2-3 Hz at all levels of the entorhinal-hippocampal loop, the only exception being the DG, where no signs of synchronized neuronal discharge could be found. Over the next $\mathbf{3 0 - 5 0} \mathrm{sec}$, the temporal organization of these EDs changed dramatically. During stage 2 , at all levels of the entorhinal-hippocampal loop, EDs occurred in 0.3-0.5 sec trains of 16-25 Hz population spikes interrupted by $0.7-1.3 \mathrm{sec}$ silent periods. The transition between stages 1 and 2 coincided with the occurrence of population spikes in the DG. Laminar analyses and multiple simultaneous field potential recordings revealed that the trains of EDs observed in stage 2 resulted from the repetitive, sequential activation of the hippocampal-entorhinal loop. In the transverse axis, the earliest event usually occurred in the CA3 region. Thereafter, population spikes occurred sequentially in the CA1 region, EC, DG, and back to the CA3 region.

Intracellular recordings confirmed that the EDs recorded extracellularly resulted from the synchronous activation of the cells in phase with the locally recorded field potentials. Dentate granule cells, layer II entorhinal cells, as well as CA1 pyramids displayed large-amplitude EPSPs crowned by

\footnotetext{
Received Sept. 23, 1991; revised Dec. 16, 1991; accepted Dec. 23, 1991.

This work was supported by NIH Grant NS13742 to R.L.; D.P. and M.D. were supported by a Canadian MRC postdoctoral fellowship and an Italian ARIN fellowship, respcctively.

Correspondence should be addressed to Rodolfo Llinás, Department of Physiology and Biophysics, New York University Medical Center, 550 First Avenue, New York, NY 10016.

Copyright (C) 1992 Society for Neuroscience $0270-6474 / 92 / 121867-15 \$ 05.00 / 0$
}

an isolated action potential phase locked to the locally recorded field potential. In contrast, the activity of CA3 pyramids consisted of typical paroxysmal depolarization shifts on which bursts of action potentials of decreasing amplitude were observed.

These results suggest that reentrant loop activity in the hippocampal-entorhinal circuit represents the central event in the functional organization of hippocampal epileptic discharges.

The past 15 years have witnessed impressive advances in our understanding of the mechanisms undcrlying cpileptic activity (Delgado-Escueta et al., 1986). Because it was recognized that the hippocampus has one of the lowest epileptogenic thresholds (Liberson et al., 1951; Garcia-Austt et al., 1954), a large proportion of the electrophysiological studies were performed on the hippocampus, more particularly, on hippocampal slices. Most of these studies focused on the interplay between intrinsic membrane properties and synaptic factors in the generation of paroxysmal depolarization shifts and on the mechanisms responsible for the synchronization and propagation of epileptic activities. In hippocampal slices treated with bicuculline or 4-aminopyridine, the CA2-CA3 region was pinpointed as the site where epileptiform bursts are generated, and the propagation speed of epileptiform activities from CA3 to CA1 was estimated as $0.1-0.5 \mathrm{~m} / \mathrm{sec}$ (see, e.g., Schwartzkroin and Prince, 1978; Wong and Traub, 1983; Voskuyl and Albus, 1985).

The problem encountered in trying to generalize these findings to the intact brain lies in the severe restrictions imposed on the synaptic circuitry by most slice preparations. Particularly important in that respect is the interruption of the reciprocal links between the hippocampus and the entorhinal cortex (EC), which constitutes the main source of afferents to the hippocampus (Ramón y Cajal, 1893). Indeed, anatomical evidence suggests that impulses arising in the EC can be channeled back to the EC through a multisynaptic pathway including the granule cells of the dentate gyrus (DG), the pyramidal cclls of the CA3 and CAl regions, as well as those of the subiculum (cf. Amaral, 1987; Amaral and Witter, 1989).

A number of investigators have attempted to study the role of the entorhinal-hippocampal loop in epileptogenesis by preparing slices that include the subicular and entorhinal cortices together with hippocampal regions. In such preparations, it was found that epileptiform events induced in the EC by low magnesium or GABA antagonists are transmitted by entorhinal axons to the DG (Stanton et al., 1986; Walther et al., 1986; Jones and Heinemann, 1988; Jones and Lambert, 1989, 1990). How- 
ever, it was found that entorhinal epileptiform activities were not modified by interrupting the links between the $\mathrm{EC}$ and the hippocampus (Jones and Heinemann, 1988). This observation suggests that the success of these commendable cfforts is limited by the difficulty of preserving the complete entorhinal-hippocampal loop in the plane of a single slice.

In principle, this closed loop could generate self-sustained reverberant oscillations that might propagate to other brain areas. In agreement with this possibility, recent observations in the behaving rat in vivo (Buzsáki, 1989) as well as in the isolated guinea pig in vitro (deCurlis et al., 1991) have shown that the hippocampal loop can give rise to a few cycles of reverberant activity at a frequency of approximately $20-40 \mathrm{~Hz}$ following high-intensity entorhinal stimulation. Moreover, in rats prepared with a fimbria-fornix lesion, a few cycles of reverberant loop activity can occur spontaneously (Buzsáki et al., 1991). Interestingly, depth recordings performed in humans with intractable epilepsies have shown that the electrographic signature of temporal lobe epilepsies is an $8-30 \mathrm{~Hz}$ rhythm whose amplitude gradually increases and eventually spreads to the amygdala as well as to cingulate and parietal regions (Delgado-Escueta and Walsh, 1983; Walsh and Delgado-Escueta, 1984).

In a recent series of experiments devoted to the organization of the entorhinal input to the hippocampal formation, we have observed that tetanic stimulation of the perforant path evokes rhythmic $15-25 \mathrm{~Hz}$ epileptiform activities in the hippocampal formation of the isolated and perfused guinea pig brain maintained in vitro. The similarities between these self-sustained afterdischarges and the oscillatory activity recorded in human temporal lobe epilepsy led us to investigate the possible role played by the close hippocampal-entorhinal loop in shaping hippocampal epileptic discharges. We choose to investigate this problem in the isolated brain in vitro preparation because it preserves the integrity of the synaptic connectivity and provides easy access to the EC, which is difficult to reach in the in vivo conditions. Epileptiform discharges (EDs), here defined as population spikes recorded from somata or dendritic layers, were induced by high-frequency entorhinal stimulation and were studied with extra- and intracellular recording methods. Laminar analyses and simultaneous recordings performed in the DG, the $\mathrm{CA} 3$ and $\mathrm{CA} 1$ region, as well as the $\mathrm{EC}$ showed that the entorhinal-hippocampal loop is rhythmically active during this form of experimental epilepsy. The similarity between the EEG rhythms observed in human temporal lobe epilepsy and in the present model of epilepsy suggests that the same synaptic mechanisms might be involved in both conditions.

\section{Materials and Methods}

The whole-brain in vitro preparation. Only a brief description of the isolated, arterially perfused in vitro preparation will be provided here since it has been described in previous works (Llinás et al., 1981, 1989; Muhlethaler and Serafin, 1990; de Curtis et al., 1991). Briefly, two cold $\left(10^{\circ} \mathrm{C}\right)$ solutions, a perfusate and a superfusate, were used for this preparation. Except for the addition of dextran $70(3 \%)$ to the perfusate, their composition was identical: $126 \mathrm{mM} \mathrm{NaCl}, 26 \mathrm{~mm} \mathrm{NaCO}_{3}, 3 \mathrm{~mm}$ $\mathrm{KCl}, 1.2 \mathrm{~mm} \mathrm{KH}_{2} \mathrm{PO}_{4}, 1.3 \mathrm{~mm} \mathrm{MgSO}_{4}, 2.4 \mathrm{~mm} \mathrm{CaCl}$, $5 \mathrm{~mm}$ HEPES, $15 \mathrm{~mm}$ dextrose, and $0.4 \mathrm{~mm}$ thiourea. Both solutions were saturated with an oxygenated gas mixture $\left(95 \% \mathrm{O}_{2}, 5 \% \mathrm{CO}_{2}\right)$.

Barbiturate-anesthetized (Somnotol; $35 \mathrm{mg} / \mathrm{kg}$ ) Hartley guinea pigs $(150-200 \mathrm{gm})$ were perfused intracardially with the cold, oxygenated perfusate. During the perfusion, an extensive craniotomy was performed, the dura mater was cut on the midline, and the hippocampus was exposed by carefully removing the overlying cerebral cortex and white matter using a suction cannula. At this point, the perfusion was interrupted, the cranial nerves and the carotid arteries sectioned, and the brain transferred to the recording chamber. Initially, the brain was positioned with its ventral surface up and a polyethylene cannula was inserted in one of the vertebral arteries. As the arterial perfusion was started, the leaking arteries were ligated. A peristaltic pump delivered the perfusate at a rate of $6-7 \mathrm{ml} / \mathrm{min}$. In addition, the brain was continuously superfused with a cold oxygenated Ringer's solution.

Once the leaking arteries were tied off, the brain was positioned with its dorsal surface facing up and the temperature of the bath and recording chamber was increased to $29-31^{\circ} \mathrm{C}$ at a rate of $0.10^{\circ} \mathrm{C} / \mathrm{min}$. A thermostat connected to a heat exchanger plate controlled the temperature of both the perfusate and superfusate. The complete sensory deafferentation and the low temperature at which these experiments were conducted preclude the possibility of discomfort for the animal.

Stimulating and recording procedures. To induce epileptic activity and identify physiologically the recording sites, two coaxial stimulating electrodes were inserted in the medial EC. Entorhinal-evoked field potentials and EDs were recorded with tungsten microelectrodes (Frederick Haer; $0.1-0.3 \mathrm{M} \Omega$ ) positioned in the CA1 and CA3 regions, the DG and/or the EC with independent micromanipulators (see Physiological identification of the recording sites, below). In order to study the septotemporal distribution of epileptic discharges, four electrodes were positioned in the CA1 region. The signals were fed into a multichannel amplifier (band pass, $0.5-3000 \mathrm{~Hz}$ ). The output of the amplifier was digitized (sampling period, $100 \mu \mathrm{sec}$ ) and stored on a magnetoscopic tape. In several experiments, wc combined field potential recordings with an intracellular analysis of hippocampal or entorhinal epileptic discharges. In these experiments, the distance between the two recording electrodes was less than $0.5 \mathrm{~mm}$ in order to study the relation between intracellular events and local field potentials. Intracellular recordings were obtained with glass micropipettes filled with $3 \mathrm{M} \mathrm{K}$-acetate solution (resistance, 50-90 M $)$ using an active bridge circuitry (Neurodata, model IR-283). The signals were digitized with a Neurodata recording instrument (model DR-484) and stored on a magnetoscopic tape. Analyses were performed off line with a digital oscilloscope.

Histological controls and morphological identification of intracellularly recorded neurons. When field potential recordings werc pcrformed, the location of the electrode tip and/or its trajectory were marked by small electrolytic lesions. At the end of the experiments in which no intracellular recordings were performed, the brains were perfused with a formaldehyde solution ( $10 \%$ in $0.9 \%$ saline), sectioned on a freezing microtome (at $80 \mu \mathrm{m}$ ), and stained with thionin or neutral red to verify the position of the recording electrodes. The microelectrode tracks were reconstructed by combining micrometer readings with the histological control.

In those experiments where intracellular recordings were performed, morphological identification of the recorded neurons was implemented by intracellular injections of biocytin. Biocytin at $2 \%$ (Sigma) was added to the electrolytic recording solution and iontophoresed into the cell with a continuous $0.5 \mathrm{nA}$ hyperpolarizing current (for $5 \mathrm{~min}$ ) superimposed to $200 \mathrm{msec}$ hyperpolarizing pulses $(0.5 \mathrm{nA}, 3 \mathrm{~Hz})$. At the end of the experiment (at least $2 \mathrm{hr}$ after the intracellular biocytin injection), the isolated brain was fixed (arterial perfusion with $10 \%$ formalin in $0.9 \%$ saline) and immersed overnight in a sucrose solution (30\% in phosphate buffer). One hundred micrometer frozen sections were attained, incubated in the avidin-biotin-HRP complex solution (ABC Elite kit, Vector) and processed to reveal the intracellular HRP staining (Horikawa and Armstrong, 1988).

\section{Results}

\section{Physiological identification of the recording sites}

The strict laminar organization of the hippocampal formation (Ramón y Cajal, 1893; Lorente de Nó, 1934) has allowed the development of unquestionable criteria to identify the recording sites. The shape of the field potentials recorded at most levels of the hippocampal formation following entorhinal stimulation has been known since the pioneering studies of Andersen and collaborators (Andersen et al., 1966a,b) and confirmed repeatedly by numerous investigators both in vivo (see, e.g., Bartesaghi et al., 1983) and in the isolated brain maintained in vitro (deCurtis 

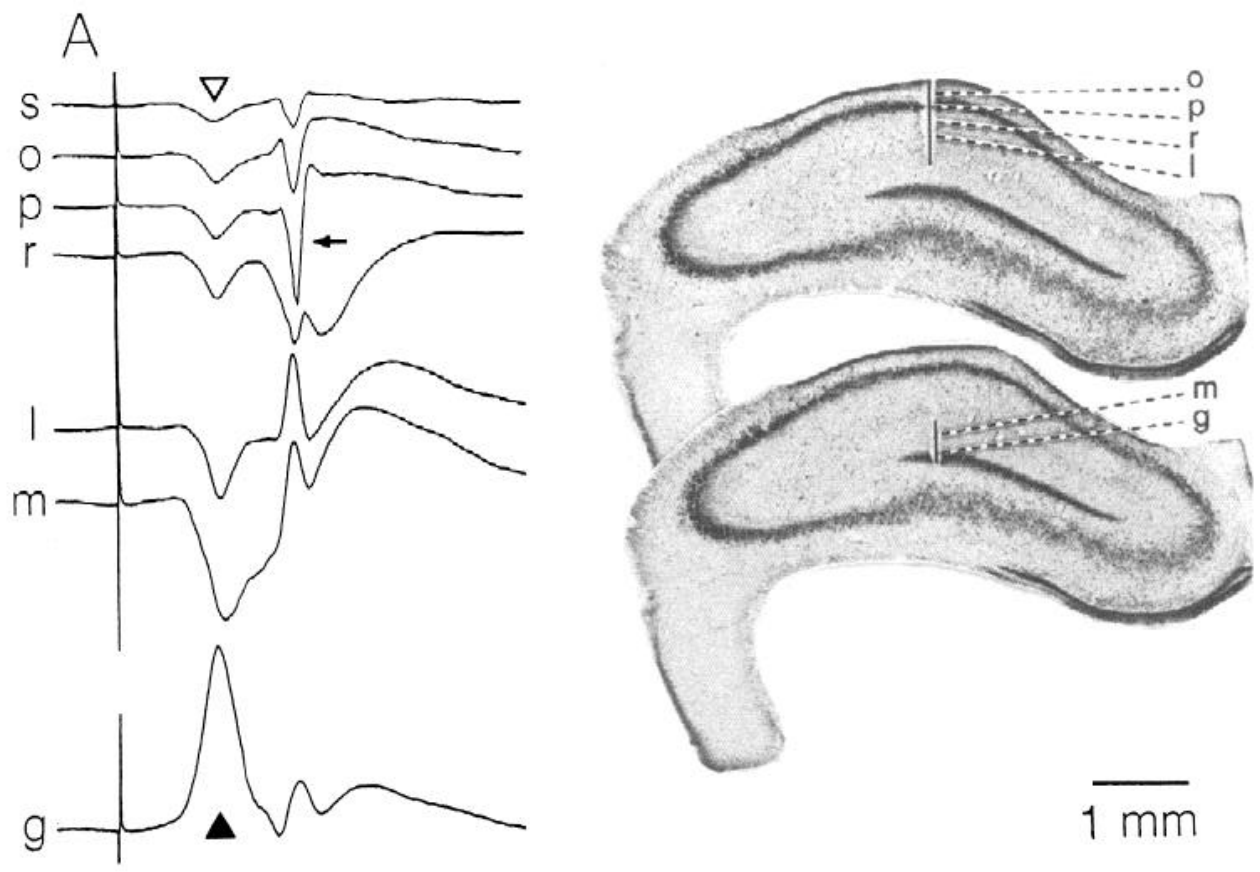

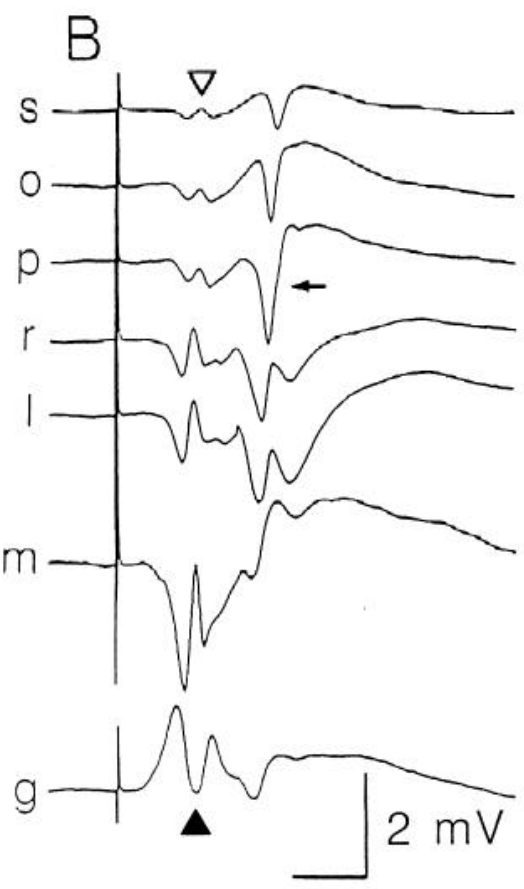

$10 \mathrm{~ms}$

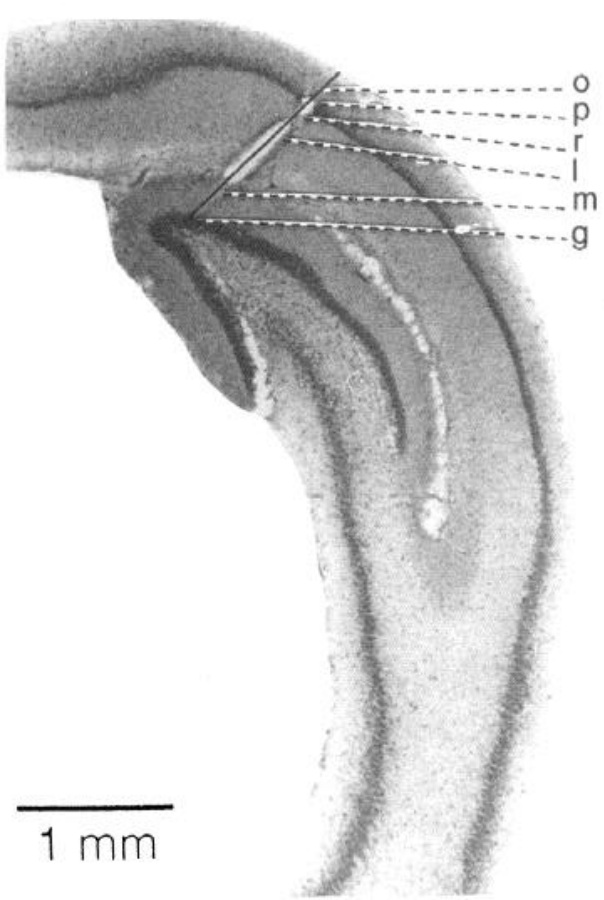

Figure 1. Laminar analysis of ECevoked field potentials in the hippocampal formation: dorsoventral tracks through the septal pole of the hippocampus $(A)$ and through a more temporal level of the hippocampus $(B)$. Both tracks were performed in $50 \mu \mathrm{m}$ steps. Each trace was obtained by averaging five EC-evoked field potentials. The solid triangles indicate DG responses as recorded in the granule layer, while the open triangles indicate the DG volumeconducted potentials recorded in the CAl region. The small arrows point to the population spike generated in the CA1 pyramidal layer. In this and following figures, positive is up. $g$, stratum granulosum of DG; $l$, stratum lacunosum of $\mathrm{CA} 1 ; m$, stratum moleculare of the DG; $o$, stratum oriens; $p$, stratum pyramidale of $\mathrm{CA} 1 ; r$, stratum radiatum; $s$, surface. et al., 1991). In the present study, the determination of the recording sites was further facilitated by the fact that the hippocampus was directly exposed. This procedure allowed precise placement of the electrodes on the surface of the hippocampus and adjustment of the angle of penetration to compensate for the curvature of the hippocampus. In all the experiments described below, the electrode position was adjusted under physiological control prior to the induction of the EDs.

Although a direct and an indirect (through the DG) entorhinal projection to the CA1 region has been described (cf. Wyss, 1981), only trisynaptic population spikes (arrows in Fig. 1; latency, $>15 \mathrm{msec}$ at $30^{\circ} \mathrm{C}$ ) were observed in the CAl region in response to stimulation of the medial EC. However, in disagreement with the lamellar hypothesis (Andersen et al., 1971), they were widely distributed in the longitudinal and transverse axes of the hippocampus. The distribution of EC-evoked population spikes in the DG was restricted in the longitudinal axis: stimulating sites located in the caudal part of the medial EC elicited population 

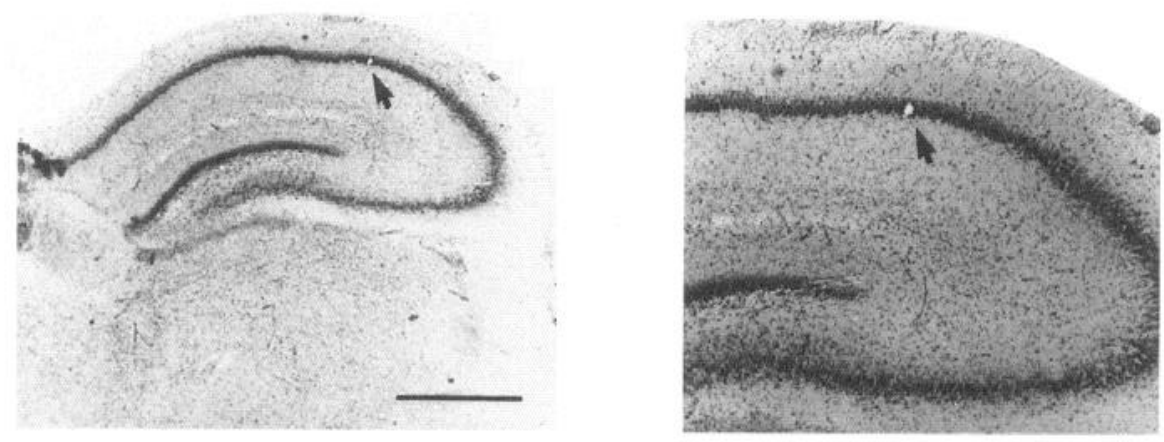

A

B
Figure 2. Evolution of epileptiform epoches elicited by high-frequency stimulation of the EC. In $A$ and $B$, the recording electrode was positioned in the pyramidal layer of the CAl region as confirmed by the histological control shown at the top (arrows point to a small electrolytic lesion). In $A$, EDs were elicited by three $1 \mathrm{sec}$ pulse trains $(100 \mathrm{~Hz})$ applied to the medial EC. The time on the left refers to the interval between the interruption of EC trains and the onset of the trace. The sequence of potentials marked by an asterisk is shown with a different time base in Figure 3. $B$ illustrates with a slower time base a short-lasting period of epileptiform activity induced by a single EC pulse train (arrowhead). Scale bar: $1 \mathrm{~mm}$ for the left photomicrograph; $0.5 \mathrm{~mm}$ for the right one.
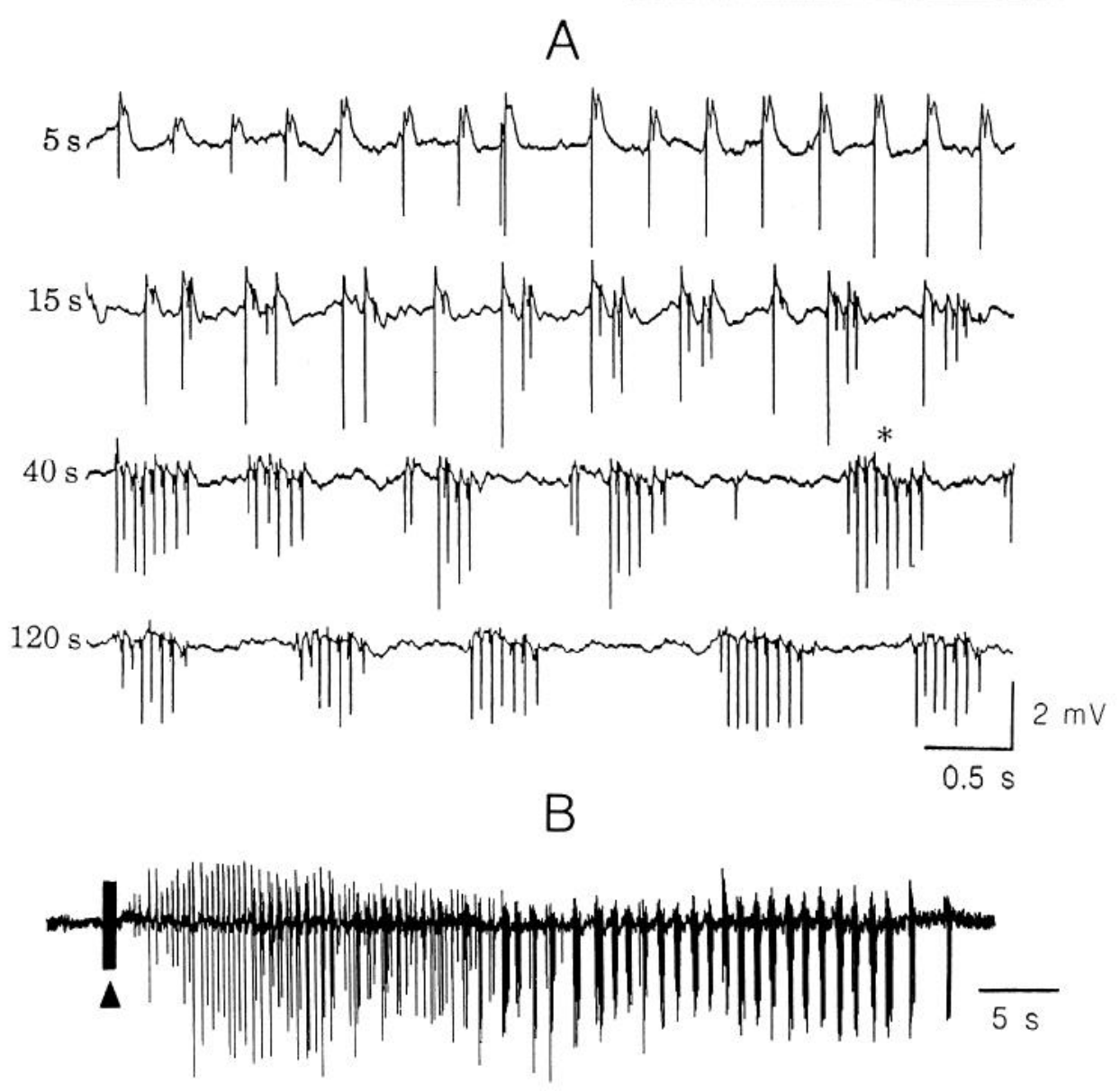

tracks, the stimulating electrode was located halfway in the rostrocaudal extent of the medial EC. Note the polarity reversal of all the components of the EC-evoked potentials during the penetration, ruling out volume conduction from distant sites. In the electrode track shown in Figure $1 A$, the entorhinal stimulation failed to evoke a population spike in the DG (solid triangle in Fig. $1 \mathrm{~A}$ ). In contrast, EC stimulation elicited a clear population spike at a more temporal level of the DG (solid triangle in Fig. $1 B$ ). Note that the reflection of DG responses in the CA1 region varies accordingly. When EC stimulation failed to evoke a population spike in the underlying segment of the DG, the volume-conducted DG response observed in $\mathrm{CA} 1$ was negative in polarity (open triangle in Fig. $1 A$ ). When EC stimulation evoked a population spike in the DG, the volume-conducted potential observed in the overlying CA1 region was negativepositive-negative in polarity (open triangle in Fig. $1 B$ ). As dis- 
cussed below, this factor probably accounts for some of the variations in the epileptiform activities recorded in the CA1 pyramidal layer.

\section{Induction of EDs by entorhinal stimulation}

EDs were induced by repetitive $1 \mathrm{sec}$ pulse trains $(100 \mathrm{~Hz}, 100$ $\mu \mathrm{sec}$ ) applied to the medial EC at a frequency of $0.5 \mathrm{~Hz}$. Typically, self-sustained EDs appeared only after three to five entorhinal pulse trains. Their time course and spatial distribution were very stereotyped, thereby allowing us to compare epileptic epoches elicited in the same or in different experiments. Usually, these periods of hypersynchronous neuronal activity lasted for 4-6 min and were followed by a period of decreased synaptic responsiveness (virtual abolition of EC-evoked responses) lasting 5-15 min. Because the temporal evolution of epileptiform activities at the different levels of the entorhinal-hippocampal loop (with the exception of the DG) were identical in most respects, we will initially describe CA1 EDs and reserve the description at other levels for the section devoted to simultaneous recordings of the CA3, CA1, DG, and EC.

Figure $2 A$ illustrates a typical epoch of EC-induced EDs recorded from the pyramidal layer of the CA1 region as confirmed by the histological control shown in the top part of the figure. EDs initially appeared as isolated population spikes occurring at a frequency of $2-3 \mathrm{~Hz}$. From this point on, the temporal organization of the epileptic discharges changed gradually. At every level of the hippocampal-entorhinal loop, EDs began to occur in population spike clusters of increasing duration. Approximately $30-50 \mathrm{sec}$ after their appearance, EDs occurred in $0.3-0.7 \mathrm{sec}$ trains of $16-20 \mathrm{~Hz}$ population spikes (Figs. $2 A, 3 A$ ) interrupted by silent periods lasting $0.7-1.3 \mathrm{sec}$. This pattern of activity usually persisted for $2-4 \mathrm{~min}$. Thereafter, the length of the population spike trains began to decrease as the silent periods grew longer and longer. In rare cases, even longer trains of population spikes (up to $10 \mathrm{sec}$ ) were observed. In the following, we will refer to the initial pattern of activity (isolated population spikes) as stage 1 and to the later pattern $(15-25 \mathrm{~Hz}$ trains of population spikes) as stage 2 . Figure $2 B$ illustrates an exceptionally short-lasting period of EC-induced epileptiform activity where the transition between stage 1 and 2 is very clear.

Close examination of the trains of population spikes observed in the CAl region revealed that, with the exception of the first, each population spike was preceded by a small-amplitude, shortlasting potential that could be negative (Fig. 3), or negativepositive-negative (Fig. 4). Although the shape and polarity of this potential varied from recording site to recording site, it was generally stereotyped at a particular recording site (compare Figs. 3, 5, and 7). In fact, the variability of this epileptiform potential is similar to that of the EC-evoked DG far field (see Physiological identification of the recording sites, above, and Fig. 1). Moreover, the interval between EC-evoked CA1 population spikes and the volume-conducted DG field was approximately $11-13 \mathrm{msec}$, compared to $16-18 \mathrm{msec}$ for the interval between the epileptiform CAl population spike and the preceding small-amplitude potential.

In order to verify if the small potential preceding the epileptiform CAl population spike was generated in the DG, we performed a laminar analysis (50 $\mu \mathrm{m}$ steps) of EC-induced EDs using a fixed $\mathrm{CAl}$ clectrode as a temporal refercnce in five different experiments (Fig. 4). In all experiments, the CA1 population spike reversed in stratum lacunosum while the smalleramplitude preceding potential reversed in stratum granulosum
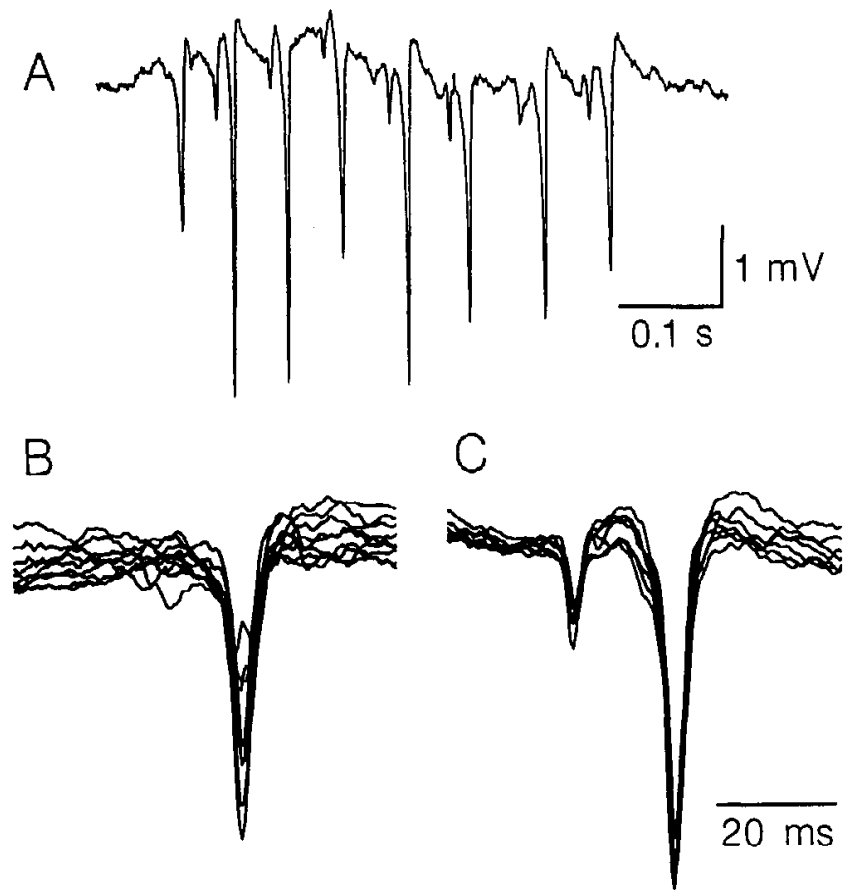

Figure 3. Comparison between the first and subsequent population spikes comprising the epileptiform sequences induced by repetitive, high-frequency stimulation of the EC: extracellular recording obtained from the pyramidal layer of the CAI region. $A$ shows the sequence of population spikes marked by an asterisk in Figure 2. Below, the first $(B)$ and subsequent $(C)$ population spikes taken from different epileptiform sequences are superposed. Note that the first population spike of an epileptiform sequence occurs in isolation while those occurring later are preceded by a small-amplitude negative potential.

of the DG. When the small-amplitude potential preceding CA1 epileptiform population spikes was negative-positive-negative in the pyramidal layer (Fig. 4), our laminar analyses always revealed the presence of a population spike in the DG $(n=3)$. Conversely, when the volume-conducted DG potential was negative in the CA1 region, our laminar analyses showed that this particular sector of the DG failed to discharge population spikes during the epileptiform epoch $(n=2)$. These results suggest that, with the exception of the first one, each CAl population spike observed during the epileptiform sequences of stage 2 results from the activation of dentate granule cells.

\section{Simultaneous recordings of $C A 3, C A 1, D G$, and $E C$}

To substantiate the above conclusion, recordings were simultaneously obtained from the layer II of the EC, the molecular layer of the DG, as well as the pyramidal layer of the regions $\mathrm{CA} 3$ and CA1 in six experiments (Fig. 5). In agreement with the results of the laminar analysis of EC-induced EDs, we observed that the population spikes occurring in the DG always coincided with the small potential preceding each $\mathrm{CAl}$ and $\mathrm{CA} 3$ population spike except the first one. However, because the electrodes were not positioned at the same septotemporal level, the variations in amplitude of the DG population spike and those of the volume-conducted DG potential recorded in CA1 and $\mathrm{CA} 3$ were often uncorrelated. Nevertheless, they were always correlated in time.

In addition, these experiments revealed that each train of population spikes observed in the $\mathrm{CAl}$ region reflects ongoing reverberant activity in the hippocampal-entorhinal loop. The 
Figure 4. Laminar analysis of ECevoked EDs: dorsoventral track through the hippocampus at mid septotemporal level in $50 \mu \mathrm{m}$ steps. A fixed electrode positioned in the pyramidal layer of the CA1 region ( $0.5 \mathrm{~mm}$ septally) was used as a temporal reference. Recordings on the left show the potentials observed during the electrode track illustrated in the camera lucida drawing to the upper right (arrow) and in the corresponding photomicrograph shown to the lower right. Note reversal of the negative population spike below the pyramidal layer and the subsequent reversal of the smallamplitude component in the granule layer of the DG. $L G$, lateral geniculate nucleus; $M G$, medial geniculate nucleus; $\operatorname{PrT}$, pretectum; $P V G$, periventricular gray matter; $S N$, substantia nigra. Other abbreviations are as in Figure 1.

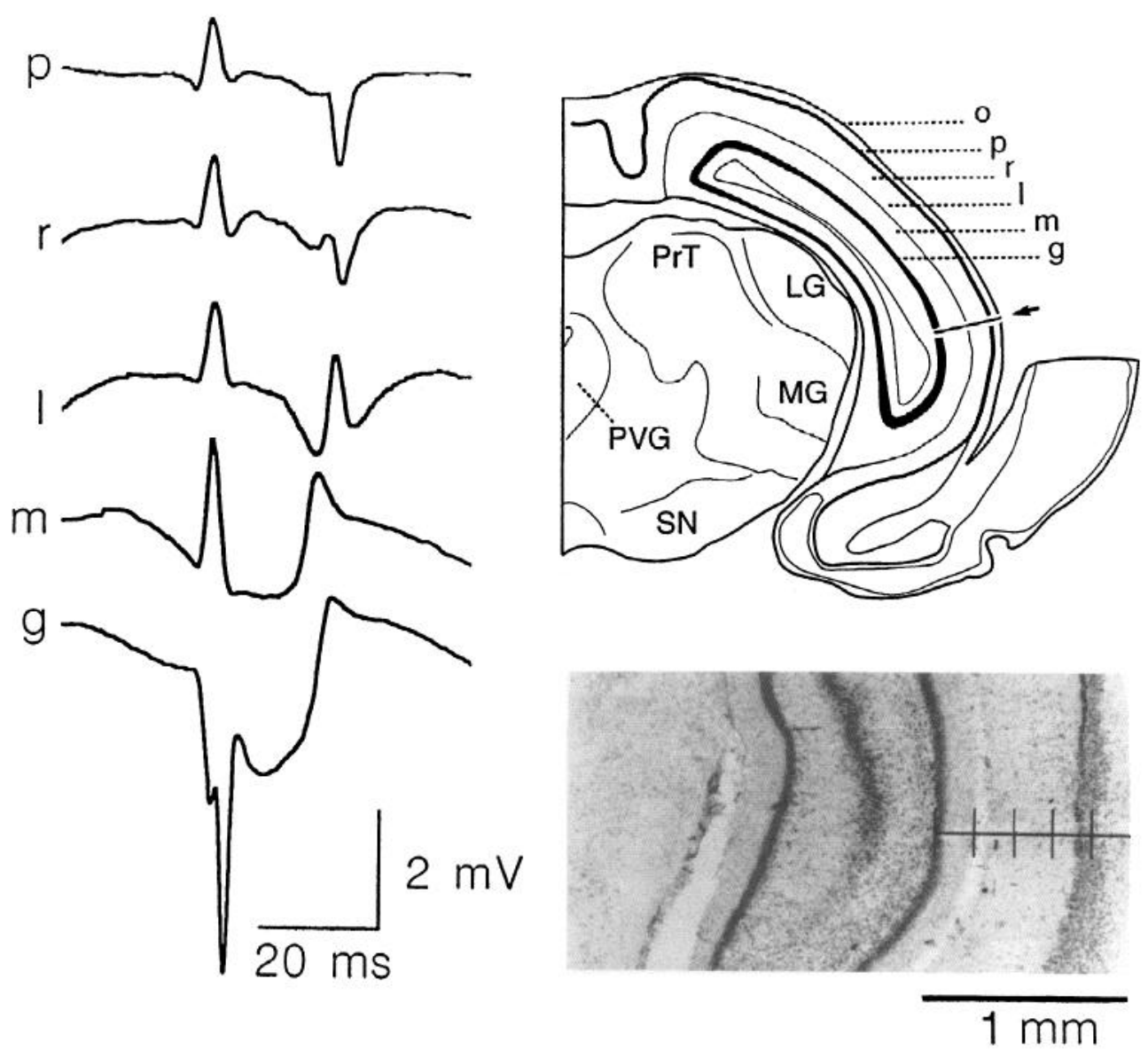

earliest population spike usually occurred in the CA3 region (asterisk in Fig. $5 B$ ) and coincided with a positivity in the molecular layer of the DG. Thereafter, population spikes occurred sequentially in the $\mathrm{CA} 1$ region, $\mathrm{EC}, \mathrm{DG}$, and the $\mathrm{CA} 3$ region. The sequential nature of the activities recorded at the different levels of the hippocampal-entorhinal loop can be seen easily in Figure $5 B$, where the first three epileptiform cycles of a population spike train are illustrated with an extended time base.

These experiments also revealed that the limiting factor determining the duration of the reverberating activity during the epileptiform trains of stage 2 is probably the EC. In contrast with the population spikes generated at the CA3, CA1, and DG levels, those recorded in the EC were usually quite labile. As a rule, the amplitude of the population spikes generated by the EC diminished rapidly. After three to four cycles, the EC usually failed to generate clear, large-amplitude population spikes. Instead, the initial large-amplitude EC population spikes were replaced by slowly rising negative potentials of smaller amplitude (Fig. 5).

\section{EDs of stage 1 and transition between stages 1 and 2}

The isolated EDs occurring in the $\mathrm{CA} 3$ and $\mathrm{CA} 1$ region during stage 1 as well as the first EDs of the epileptic sequences characteristic of stage 2 had a different origin than those occurring later in these trains of EDs. In contrast with the latter, they were never preceded by a volume-conducted DG potential, thus suggesting that they were not caused by a mossy fiber input. In agreement with this view, field potential recordings obtained from the granular layer of the DG revealed that dentate granule cells failed to generate population spikes during stage 1 (Fig. $6 A 1$ ) but did so during stage 2 (Fig. $6 A 2$ ).

Simultaneous recordings of the different links of the entorhinal-hippocampal loop revealed that the EDs of stage 1 were initiated in the CA3 region, propagated to the CA1 sector, and from there to the EC. However, the ensuing return volley from the EC failed to evoke a population spike in the DG (Fig. 6BI). As a result, the EDs recorded in regions CA1 and CA3 during stage 1 occurred in isolation, at a frequency of $2-3 \mathrm{~Hz}$. In six different experiments, we observed that the transition from the isolated CA3-CA1 population spikes characteristic of stage 1 to the rhythmic $15-25 \mathrm{~Hz}$ EDs of stage 2 coincided with the appearance of the first population spike in the DG. This is illustrated in Figure $6 \mathrm{~B}$, which shows field potential recordings obtained from the pyramidal layer of the $C A 3$ region, the layer II of the EC, and the granule layer of the DG, before (Fig. 6BI) and during (Fig. 6B2) the transition from stage 1 to stage 2 . Note that reactivation of the CA3 region occurred only when the DG generated a population spike in response to the perforant path input.

\section{Septotemporal organization of EC-induced EDs}

To gain some insight in the longitudinal organization of hippocampal activities during EC-induced EDs, in five experiments we performed multiple simultaneous recordings of the CAl pyramidal layer at different septotemporal levels (Fig. 7). These experiments revealed that the vast majority of the EDs observed 

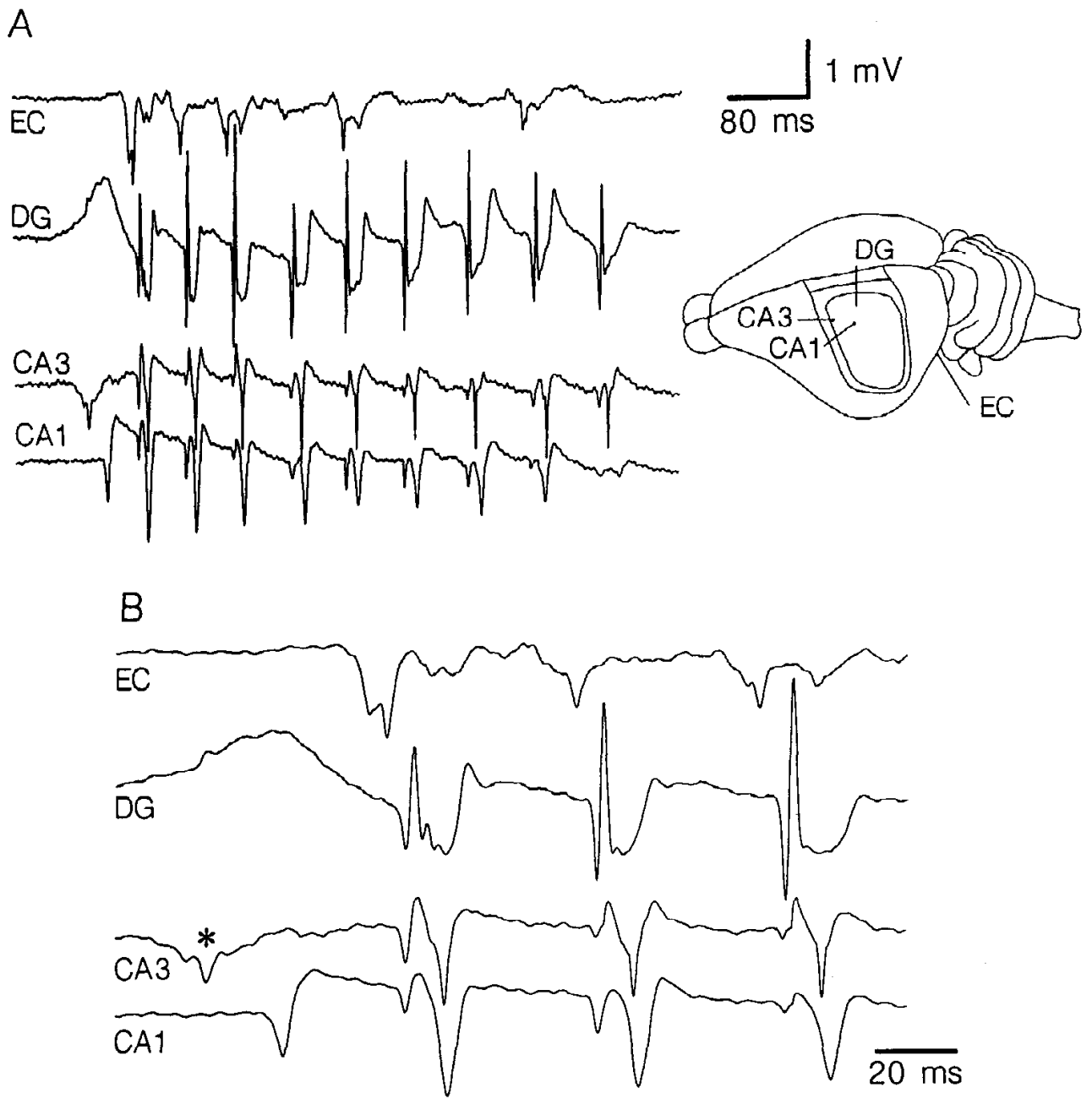

Figure 5. Sequential activation of the entorhinal-hippocampal loop during EC-induced EDs: simultaneous field recordings obtained from the layer II of the EC, molecular layer of the DG, and pyramidal layer of regions $\mathrm{CAl}$ and CA3. $B$ illustrates, with a more extended time base, the onset on the sequence of potentials shown in $A$. The approximate position of the recording electrodes is illustrated on the scheme shown on the right. Note that the earliest event occurs in the CA3 region.

in stage 1 and 2 arose at temporal levels of the hippocampal formation. This phenomenon is illustrated in Figure 7, $A$ and $B$, where four CAl electrodes were placed at $1 \mathrm{~mm}$ intervals. Note that the earliest event is a slowly rising negativity that appears first at the recording site located more temporally and then spreads septally with a conduction velocity of approximately $0.1-0.2 \mathrm{~m} / \mathrm{sec}$. Epileptiform sequences having a septal origin were observed exceptionally (Fig. 7C).

Another interesting observation arising from these experiments is that during EC-induced epileptiform sequences, the activity of the hippocampus at different longitudinal levels undergoes a progressive synchronization. This can be best understood by drawing imaginary lines between the peak of the most septal and the most temporal CA1 population spikes (Fig. $7 \mathrm{~A}$ ) and by repeating this operation for each epileptiform cycle. It is clear that after a few cycles, these initially oblique lines become virtually vertical, a reflection of the progressive synchronization of CA1 activities. In parallel with this process, note the gradual increase in the amplitude of the positive DG far field in the recording sites located more septally (Fig. $7 A$ ), which probably reflects the increasingly synchronous activation of DG. The small amplitude of the volume-conducted DG potential in traces 3 and 4 of Figure 7 should not be interpreted as evidence of a lack of activation of the DG at more temporal levels of the hippocampus. This phenomenon simply reflects the fact that the distance between these recording sites and the DG is larger because of the caudally directed curvature of the DG.

\section{Intracellular recordings of epileptiform activities}

To establish that the EDs recorded extracellularly at the different levels of the hippocampal-enthorinal loop result from the synchronous activation of the cells in phase with the locally recorded field potentials, intracellular recordings of dentate granule cells $(n=7)$, entorhinal layer II $(n=6)$ and V $(n=4)$ neurons, as well as CA $1(n=8)$ and CA3 $(n=7)$ pyramids were performed. These neurons had a resting membrane potential higher than $-65 \mathrm{mV}$ and overshooting action potentials. The identity of these various types of neurons was ascertained by morphological (biocytin injections; see Fig. 10) and/or electrophysiological criteria. For instance, CA3 pyramids were identified by their propensity to discharge high-frequency (100-150 $\mathrm{Hz}$ ) spike bursts in response to depolarizing current pulses or high-intensity EC stimuli, confirming similar findings in the slice by Wong and Prince (1978). By contrast, as described by Schwartzkroin (1977), CAl pyramids discharged slower accommodating trains of action potentials in response to intracellularly injected depolarizing current pulses. These two types of cells were distinguished from non-pyramidal cell types by their lower spontaneous activity and longer-duration action potential (Schwartzkroin and Mathers, 1978; Lacaille et al., 1989). Fi- 

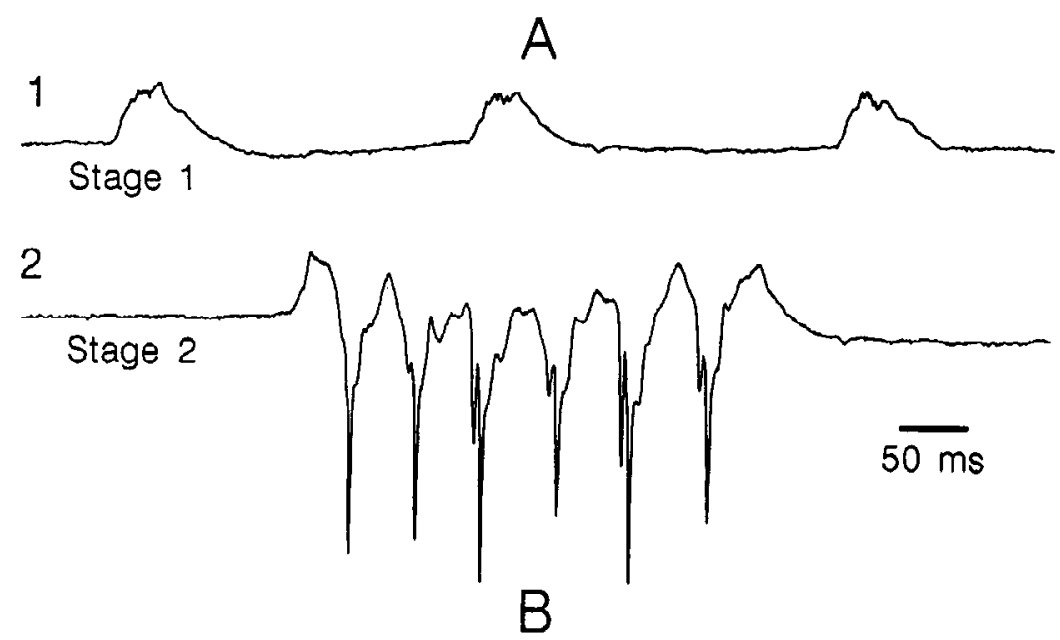

Figure 6. Field potential recordings of DG activity during EC-induced EDs. In $A$, the electrode tip was in the stratum granulosum. In $B$, field potential recordings were simultaneously obtained from the pyramidal layer of the $\mathrm{CA} 3$ region, layer II of the $\mathrm{EC}$, and stratum granulosum of the DG.
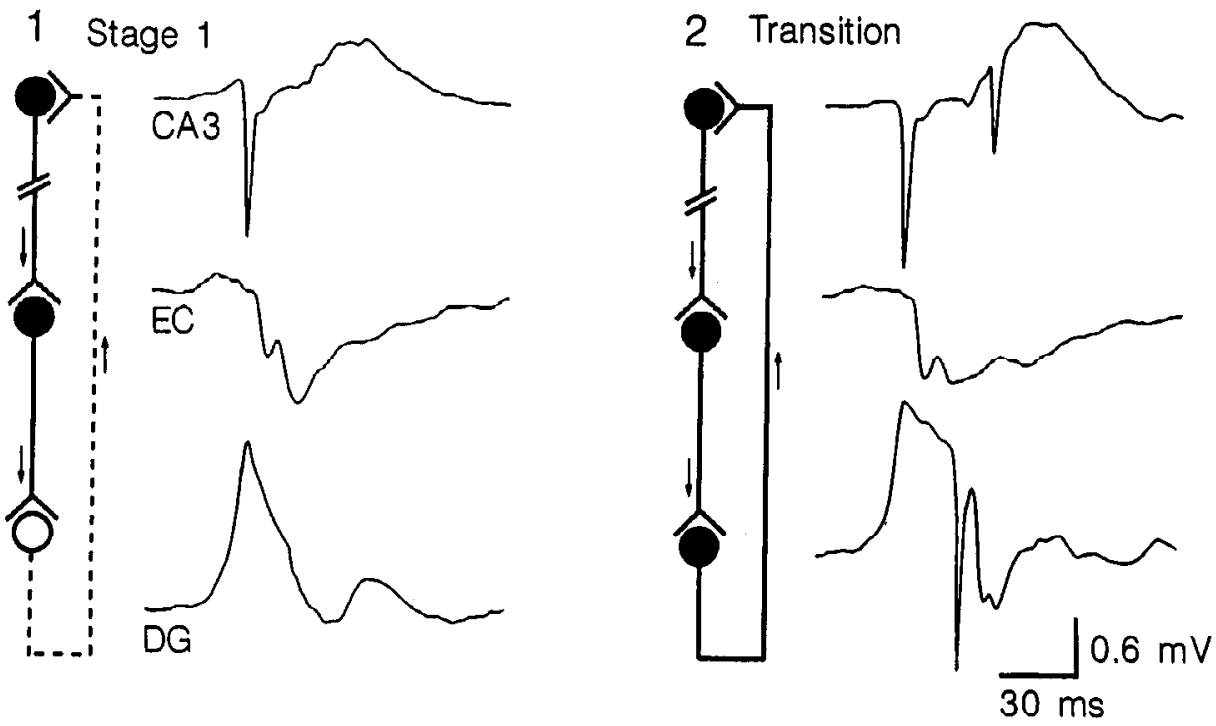

nally, we verified the laminar location of the recorded neurons by inspecting the shape of the EC-evoked field potential at the end of the intracellular recording.

These intracellular recordings confirmed that dentate granule cells, layer II and V entorhinal neurons, as well as CAl pyramids display large-amplitude depolarizations crowned by an isolated action potential phase locked to the population spikes present in the locally recorded field potential. Although no specific tests have been performed at this time regarding the ionic mechanisms responsible for the generation of these potentials, it is likely that they result from the activation of both ligand- and voltage-activated conductances. For simplicity, in the following, we will refer to these depolarizations as EPSPs.

The case of a CA1 pyramid is illustrated in Figure $8 \mathrm{~A}$. An exceptionally long sequence of EDs occurred while we were recording this neuron. Note the action potentials and underlying large-amplitude EPSPs (7-11 mV) that occurred in phase with each population spike at a frequency of $15 \mathrm{~Hz}$. As in most cells, the first EPSPs were larger than those occurring later in the epileptiform sequence. In addition, note that the membrane potential did not return to resting values between each EPSPs, another phenomenon common to all cells. Instead, the cell remained tonically depolarized by $6-8 \mathrm{mV}$. This sustained de- polarization was accompanied by a gradual decrease in the amplitude of the action potential.

Figure $8 B$ shows the epileptiform activity of a typical entorhinal stellate cell that was identified morphologically, by intracellular biocytin injection, and physiologically, by the presence of high-frequency voltage-dependent oscillations (Alonso and Llinás, 1989; deCurtis et al., 1991). The left panel of Figure $8 B$ shows the activity of the cell during the transition between stage 1 and stage 2, while the right panel was obtained after establishment of stage 2. During both stages, the cell displayed largeamplitude EPSPs that triggered action potentials in phase with the population spikes present in the simultaneously recorded local field potential. Comparison of the membrane potential before and $3 \mathrm{~min}$ after the induction of the epileptiform epoch revealed that the cell underwent a progressive depolarization reaching up to $10 \mathrm{mV}$ during this period (compare the left and right panels in Fig. $8 B$ ). This phenomenon was observed in the different types of cells we recorded along the hippocampalentorhinal loop. A similar pattern of activity was seen in morphologically identified layer $\mathrm{V}$ pyramidal cells of the EC (see Fig. 10A).

Like the other links of the hippocampal-entorhinal loop, CA3 pyramids displayed large-amplitude EPSPs phase locked to the 

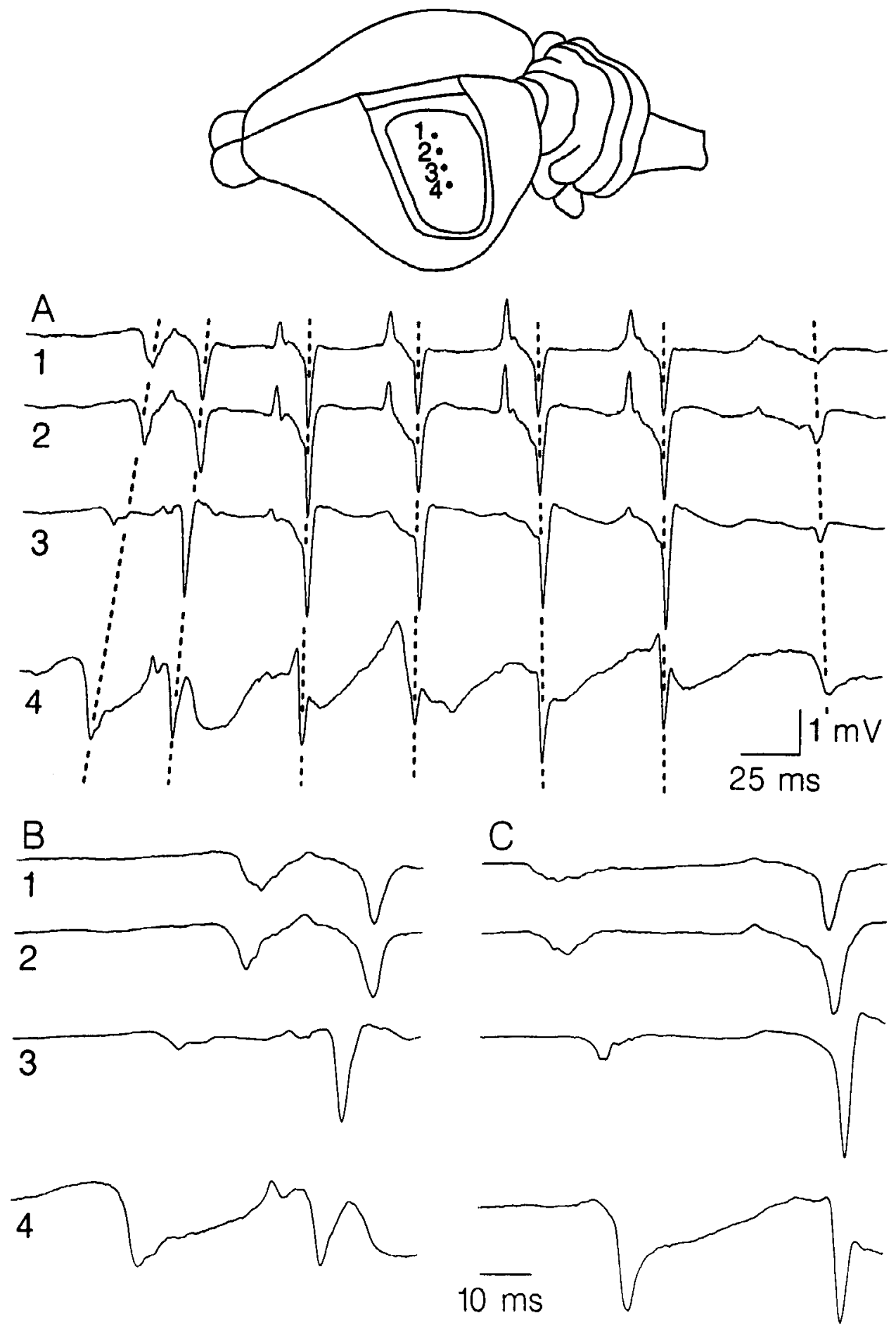

Figure 7. Origin and synchronization of epileptiform activities in the longitudinal axis of the hippocampus: four simultaneous field recordings of the CA1 pyramidal layer at different septotemporal levels. The drawing at the top shows the approximate location of the recording electrodes. $B$ shows with an extended time base the onset of the sequence of potentials shown in $A$. $C$ depicts another epileptiform sequence. Note the progressive synchronization of the population spikes at the different septotemporal levels.

population spikes present in the locally recorded field potential. While these EPSPs evoked isolated action potentials in stage 1, they elicited high-frequency spike bursts $(100-150 \mathrm{~Hz})$ in stage 2. Moreover, in two out of seven CA3 pyramids, the first highfrequency burst coincided exactly with the transition from stage 1 to stage 2 .

This point is illustrated in Figure 9, where the activity of a CA3 pyramid in stage 1 (Fig. $9 A$ ) and during the transition from stage 1 to stage 2 (Fig. $9 B$ ) is shown. In this particular case, the transition between stage 1 and stage 2 was very short: the only epileptiform sequence comprising less than four and more than one population spike is shown in Figure $9 B$. The first highfrequency spike burst discharged by this $\mathrm{CA} 3$ pyramid coincided with the second population spike of this particular transitional epileptiform sequence (Fig. $9 B$ ). Figure $9 C$ depicts, with an extended base, the activity of the same pyramidal cell during the population spikes marked by an asterisk in Figure $9, A$ and $B$ (see Fig. 10B for the morphological identification of this cell).

\section{Discussion}

Understanding the mechanisms underlying the distinctive pattern of neuronal activity present in the different epileptic syn- 


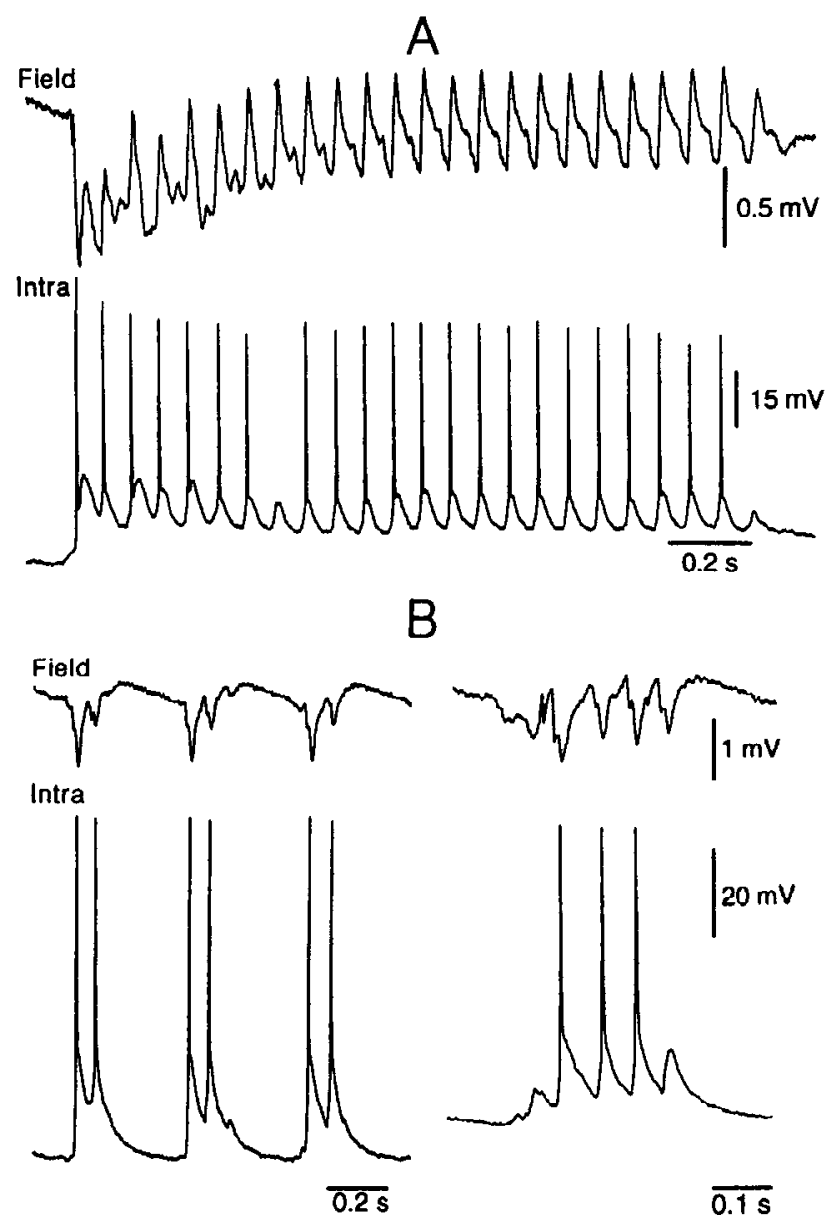

Figure 8. Correlation between intra- and extracellularly recorded epileptiform potentials in the CA1 region $(A)$ and $\mathrm{EC}(B)$. $A$ : Upper trace, Field potential recording obtained from the pyramidal layer of the $\mathrm{CAl}$ region. Lower trace, Simultancously rccorded activity of a CAl pyramid located at $400 \mu \mathrm{m}$ of the tungsten microelectrode used to record field potentials. The membrane potential of this cell before EC trains was $-72 \mathrm{mV}$. $B$, Intracellular recording of a layer II stellate cell during ECinduced EDs. The upper trace is a locally recorded field potential. The left panel shows the EDs that occurred $30 \mathrm{sec}$ after the EC trains, while the right panel shows EDs that occurred $3 \mathrm{~min}$ after the EC trains. The membrane potential of this cell before EC trains was $-69 \mathrm{mV}$.

dromes is one of the basic challenges in epilepsy research. In this context, the present series of experiments constitute an attempt to identify the functional steps generating the peculiar 8-30 $\mathrm{Hz}$ rhythm characterizing stereoelectroencephalographic hippocampal recordings in human temporal lobe epilepsy (Delgado-Escueta and Walsh, 1983; Walsh and Delgado-Escueta, 1984). We addressed this issue in the whole guinea pig brain maintained in vitro where tetanic stimulation of the EC elicits self-sustained 15-25 Hz EDs at every level of the hippocampalentorhinal loop.

The interest of this new experimental model resides in the similarities existing between the EDs observed in the present preparation and in human temporal lobe epilepsy. In spite of the fact that the precipitating factors may be quite different in the two conditions, we believe that understanding the mechanisms responsible for the EDs observed in this experimental model has important implications for clinical and fundamental research on temporal lobe epilepsy as well as normal hippocampal physiology. Here, we will discuss the main findings of the present study in light of previous anatomical and physiological results and comment on their significance for normal and pathological hippocampal function.

\section{Role of the hippocampal-entorhinal loop in the generation of the 15-25 Hz EDs}

The main finding of the present study is that the stereotyped 15-25 Hz EDs observed in the EC, DG, CA3, and CA 1 regions during stage 2 probably result from the repetitive, sequential activation of the different links of the hippocampal-entorhinal loop throughout the septotemporal extent of the hippocampal formation. As will be argued below, although this conclusion is based on correlational electrophysiological evidence, it is the most likely interpretation compatible with present knowledge regarding the synaptic architecture of the hippocampal network.

Electrophysiological studies performed in the late 1960s (Andersen et al., 1966a,b; Andersen, 1975) confirmed the intuitions of early anatomists concerning the organization of intrahippocampal connections (Ramón y Cajal, 1893; Lorente de Nó, 1933, 1934) by showing that stimulation of the EC or perforant path fibers provokes the sequential activation of dentate granule cells, CA 3 and then CA1 pyramids. It was found that the synaptic excitation of the different subfields generates large-amplitude population spikes (up to $5 \mathrm{mV}$ ) that are negative in polarity when recorded in the layer of the cell bodies. These brief negative field potentials represent the summed extracellular currents generated by the synchronous discharge of the synaptically activated neurons. Although it was originally hypothesized that the invasion of the trisynaptic circuit following EC stimulation travels along relatively narrow transverse slices (Andersen et al., 1971), subsequent anatomical (reviewed in Amaral and Witter, 1989) and physiological studies (Bartesaghi et al., 1983; Buzsáki et al., 1990; Paré and Llinás, 1991) showed that the hippocampal network can generate other patterns of activity.

In the present series of experiments, two lines of evidence suggest that the $15-25 \mathrm{~Hz}$ rhythm results from the repetitive sequential activation of the various links of the entorhinohippocampal pathway. First, the laminar profile of the epileptiform field potentials recorded in the hippocampus during stage 2 was similar to that of EC-evoked field potential responses. For instance, with the exception of the initial event, the rhythmic negative population spikes recorded in the pyramidal layer of the CAl region during stage 2 were reliably preceded by a volume-conducted DG potential. When the electrode was lowered farther in the hippocampus, the CA1 population spike reversed in polarity in stratum lacunosum as the DG potential grew in amplitude. Moreover, when the electrode was moved from the stratum moleculare to the stratum granulosum of the DG, DG potentials also reversed in polarity.

Second, when electrodes were positioned in the granular layer of the DG, the pyramidal layer of the $\mathrm{CA} 3$ and $\mathrm{CA} 1$ regions, as well as in layer II of the EC, it became clear that the EDs recorded in these different regions did not occur randomly but respected the unidirectional and sequential nature of intrahippocampal connections (Ramón y Cajal, 1893; Lorente de Nó, $1933,1934)$. The first ED usually appeared in the CA3 region and was then followed by population spikes in the CA1, EC, and DG. Thereafter, the sequential activation of the entorhinohippocampal loop continued for up to $10 \mathrm{sec}$. Intracellular recordings confirmed that the EDs recorded extracellularly resulted from the synchronous activation of the cells in phase with the locally recorded field potentials. Layer II and V entorhinal 

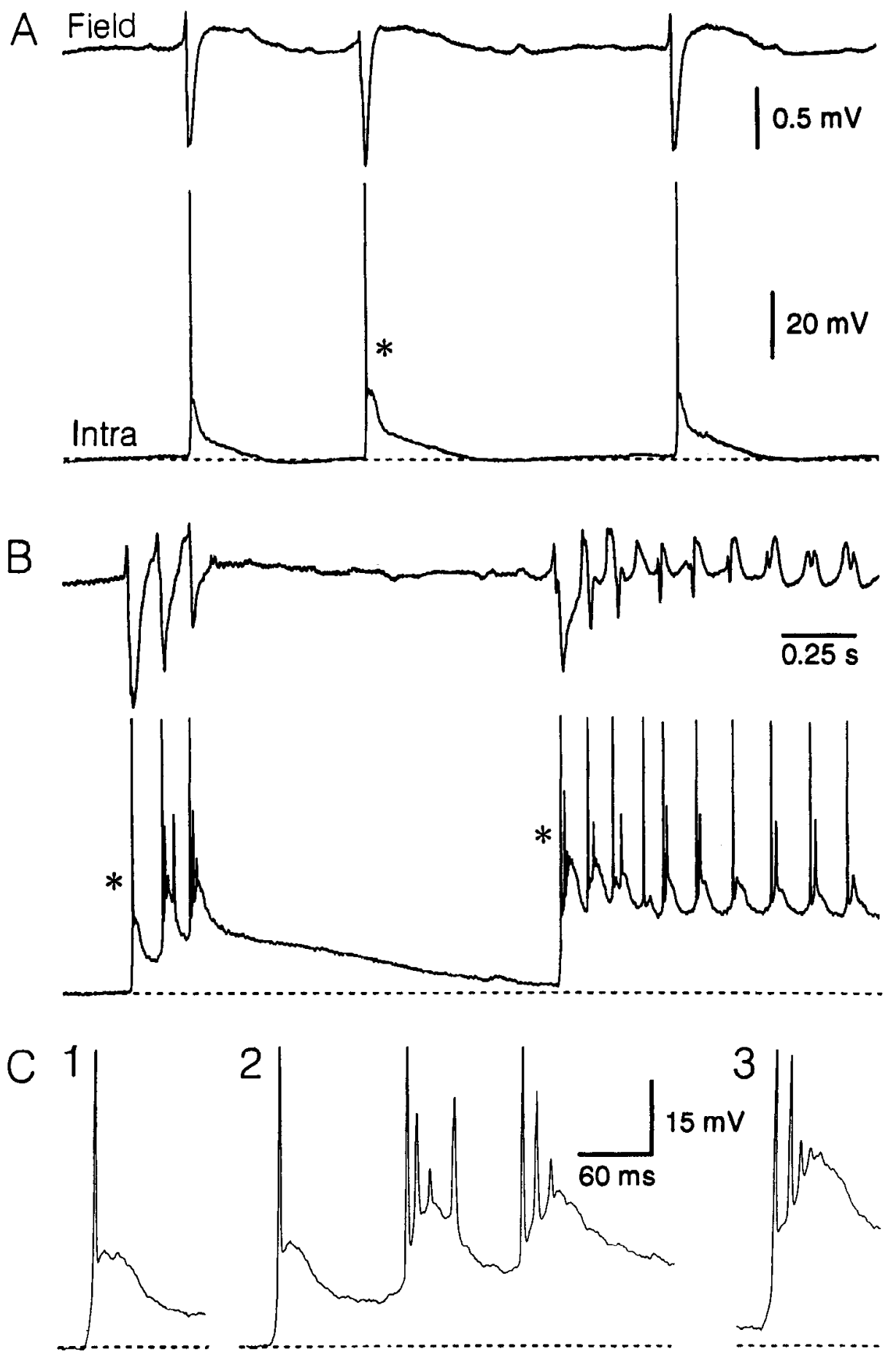

Figure 9. Intracellular recording of a pyramidal cell of the $\mathrm{CA} 3$ region during EC-induced EDs. $A$, Stage 1. $B$, Transition between stage 1 and stage. 2. $C$ illustrates, with an extended time base, the intracellular events marked by asterisks in $A$ and $B$. The membrane potential of this cell before the EC trains (base line) was $-73 \mathrm{mV}$.

cells, CA1 pyramids, as well as dentate granule cells displayed large-amplitude EPSPs crowned by isolated action potentials. In contrast, the activity of $\mathrm{CA} 3$ pyramids consisted of typical paroxysmal depolarization shifts on which bursts of action potentials were observed. Because of their propensity to burst (Wong and Prince, 1978; present results), CA3 pyramids may constitute a loop gain system that would tend to reinforce the tendency of the entorhinal-hippocampal circuit to resonnate.

Of course, an alternative interpretation for these data is that the EDs resulted from rhythmic resonant activity in the reciprocal connections between both hippocampi or between the hip- pocampal formation and another afferent structure. However, functional inactivation of hippocampal commissural connections by interrupting the perfusion of the opposite hemisphere did not prevent the appearance of the $16-25 \mathrm{~Hz}$ epileptiform rhythm. However, it is possible that amygdalohippocampal connections reinforce the propensity of the entorhinal-hippocampal circuit to be rhythmically active. Indeed, two components of the hippocampal formation, the EC and the subicular complex, are reciprocally connected to the amygdala (cf. Witter et al., 1989). Besides, this idea is consistent with evidence obtained during stereoelectroencephalographic recordings of patients af- 
Figure 10. Morphological identification of a pyramidal cell located in layer $\mathrm{V}$ of the $\mathrm{EC}(A)$ and a $\mathrm{CA} 3$ pyramid $(B)$ following intracellular injections of biocytin. Scale bar: $100 \mu \mathrm{m}$ for $A ; 70 \mu \mathrm{m}$ for $B$.

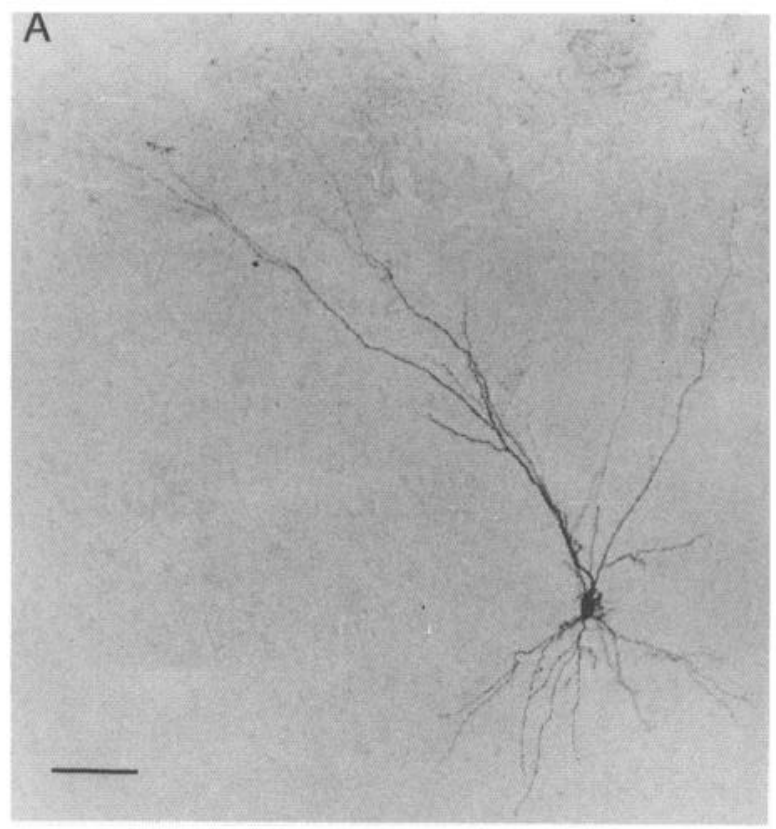

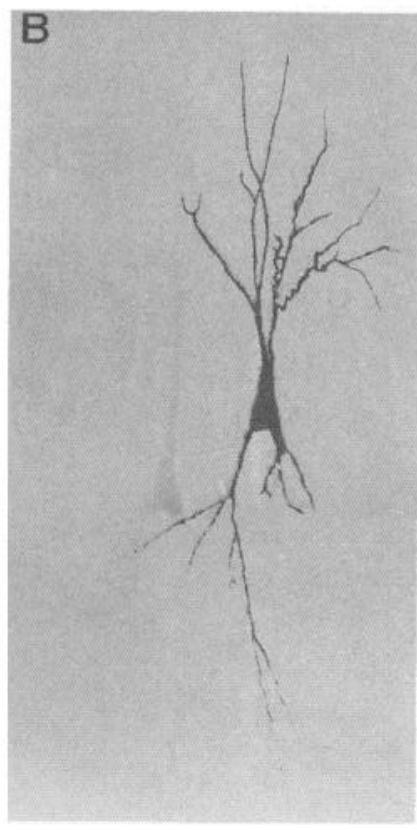

flicted with temporal lobe epilepsy. In a number of patients, the characteristic 8-30 Hz oscillation also appeared in the amygdala (Delgado-Escueta and Walsh, 1983; Walsh and Delgado-Escueta, 1984).

\section{Mechanisms through which high-frequency EC stimulation promotes epileptiform activity}

Insights in the genesis of the epileptiform activities observed in the present series of experiments come from the consideration of anatomical and physiological features of the hippocampal formation. The axons of CA 3 pyramids are highly collateralized and contribute to a prominent system of associative connections that spans across the entire septotemporal and transverse extent of the fields CA3 and CA1 (Swanson et al., 1978; Ishizuka et al., 1990). The divergent nature of these intrahippocampal associative connections endows the hippocampus with the ability to synchronize the activity of its constitutive cells as evidenced by the spontaneous occurrence of large-amplitude negative field potentials (up to $3 \mathrm{mV}$ ) in the various hippocampal subfields during slow-wave sleep and consummatory behaviors in freely moving rats (Buzsáki et al., 1983; Buzsáki, 1986; Suzuki and Smith, 1987).

However, the excitability of the hippocampal network, and thus the expression of this synchronizing mechanism, is regulated by subcortical inputs coursing in the fimbria (Buzsáki, 1989) and GABAergic interneurons disseminated in the various hippocampal subfields (see Lacaille et al., 1989). In recent years, it has been found that the intrahippocampal inhibitory network is subjected to several regulatory mechanisms that contribute to the reduction of the inhibitory pressures impinging on pyramidal cells during periods of intense stimulation: (1) a presynaptic inhibition of GABA release mediated by $\mathrm{GABA}_{\mathrm{B}}$ receptors (Thompson and Gahwiler, 1989), (2) a "desensitization" of pyramidal cells' response to GABA during prolonged iontophoresis of the neurotransmitter (Wong and Watkins, 1982), and (3) the habituation of basket cells' responses during repeated stimulation (Finch and Babb, 1977).

These mechanisms may underlie the epileptogenic effects of high-frequency EC stimulation observed in the present series of experiments. In fact, this interpretation is consistent with our own observations showing that brief, repeitive high-frequency EC trains gradually diminished and eventually suppressed the IPSPs evoked by single EC testing stimuli (D. Paré, M. deCurtis, and R. R. Llinás, unpublished observations) and those of others who reported that tetanic stimuli applied to hippocampal slices induce a long-lasting depression of IPSPs (Miles and Wong, 1987; Stelzer et al., 1987).

In addition, it is possible that repetitive activation of the entorhinal-hippocampal loop by EC stimuli has provoked an increase in the extracellular concentration of potassium and a consequent shift of the reversal potential of $\mathrm{Cl}^{-}$-mediated IPSPs toward the resting potential. Bathing of hippocampal slices in high-potassium media is known to induce epileptiform activities (see, e.g., Rutecki et al., 1985; Bragdon et al., 1986). Recordings with ion-selective electrodes will have to be performed to settle this issue.

Finally, it is likely that EC trains induced a potentiation of synaptic responses at one or many levels of the entorhinalhippocampal loop (Teyler and DiScenna, 1987; Madison et al., 1991), thus increasing the likelihood that spontaneous neuronal events will synchronize interconnected neurons and lead to a propagating population event.

\section{Origin of the EDs of stage 1 and transition to stage 2}

In agreement with the conclusions of previous in vitro studies of hippocampal slices bathed in a low-magnesium medium or treated with bicuculline or 4-aminopyridine (Schwartzkroin and Prince, 1978; Wong and Traub, 1983; Voskuyl and Albus, 1985; Mody et al., 1987), we found that the isolated EDs of stage 1 originated in the CA 3 region. Through the associative pathways linking CA3 and CA1 sectors, it is likely that spontaneously active CA3 cells recruited an important number of pyramidal cells in large population events that rapidly propagated along the transverse and septotemporal axis. In agreement with this interpretation, computer simulation of transverse hippocampal slices indicates that spontaneous burst activity in one or two 
pyramidal cells is sufficient to generate a population event involving a significant proportion of pyramidal neurons through spread of excitation via divergent "associative" connections (Traub et al., 1987).

Interestingly, the epileptiform population events occurring during stage 1 did not lead to the repetitive activation of the hippocampal-entorhinal loop. Simultaneous field potential recordings obtaincd from the different links of the loop revealed that the excitatory volley generated in the $\mathrm{CA} 3$ region was transmitted to the $\mathrm{CAl}$ field and from there to the EC. However, the ensuing return volley from the EC failed to evoke a population spike in the DG. As a result, the EDs recorded in regions CAI and CA3 during stage 1 occurred in isolation, at a frequency of 2-3 Hz. Transition from the isolated CA3-CAl population spikes characteristic of stage 1 to the rhythmic $15-25 \mathrm{~Hz}$ EDs of stage 2 coincided with the appearance of the first population spike in the DG. Thus, in the present model of temporal lobe epilepsy, the DG is the determining factor in the transition from "interictal" to "ictal" epileptiform activity.

These results are consistent with the findings of Stringer, Williamson, and Lothman on the critical influence exerted by the DG on the development of kindled seizures in the hippocampus (Stringer and Lothman, 1989, 1990; Stringer et al., 1989, 1991). In urethanc-anesthetized rats, they have identified an electrophysiological event (termed "maximal dentate activation") that coincides with the lengthening of afterdischarges induced by kindling hippocampal stimulation. This event was defined as the appearance of large-amplitude population spikes in the DG granule layer associated with a secondary rise in the extracellular concentration of potassium and a prolonged negative DC shift (Stringer and Lothman, 1989, 1990; Stringer et al., 1989). In unanesthetized rats, this event was found to be preceded by another type of discharge consisting of "irregular broad positive potentials without population spikes" that "readily evolved into maximal dentate activation" (Stringer et al., 1991, p 341). It is tempting to speculate that this initial type of afterdischarge corresponds to our stage 1 while maximal dentate activation would correspond to stage 2 .

\section{Septotemporal propagation and synchronization of epileptiform activities}

Multiple simultaneous field potential recordings of the CA1 region were obtained at different septotemporal levels of the hippocampus. These recordings revealed that the majority of the EDs present in stage 1 and 2 originated from temporal levels of the hippocampus and propagated septally at a velocity of $0.1-$ $0.2 \mathrm{~m} / \mathrm{sec}$. The greater propensity of the temporal part of the hippocampus observed in the present preparation is consistent with other experimental observations obtained in various in vitro and in vivo models of epilepsy (see, e.g., Lothman and Collins, 1981; Yoshida, 1984; Gilbert et al., 1985; Bragdon et al., 1986; Lee et al., 1990). However, the mechanisms responsible for this greater susceptibility of the ventral hippocampus remain unknown (see extensive discussion on this topic by Lee et al., 1990).

In addition, our multiple simultaneous field potential recordings of the CA 1 region provided further evidence of the powerful synchronizing influence exerted by intrahippocampal associative pathways on hippocampal activities. Indeed, we observed that after completion of two or three cycles within the hippocampal-entorhinal loop, CA1 EDs became almost perfectly synchronized. In addition to the associative pathways mentioned earlier, other features of the hippocampal circuitry probably contribute to this synchronizing process. First, the different septotemporal levels of the DG are linked by a prominent system of associative connections (Swanson et al., 1978) that arise from cells of the polymorphic layer of the DG (Laurberg, 1979; Laurberg and Sorensen, 1981). Second, the entorhinal projection to the DG exhibits a certain degree of divergence (Wyss, 1981; Amaral and Witter, 1989). Third, a significant proportion of CA3-CA 1 pyramids as well as dentate granule neurons are electrotonically coupled by gap junctions (Schmalbruch and Jahnsen, 1981; MacVicar and Dudek, 1982).

\section{Implications for future research on temporal lobe epilepsy}

In light of the present findings, one of the most urgent issues to be addressed is that of determining whether the stereotypical 8-30 Hz rhythm characterizing human temporal lobe epilepsy is also generated by reverberant activity in the entorhinohippocampal loop. In addition, a number of issues should be investigated in the present preparation. First, the ligand- and voltage-activated conductances involved in the genesis of the epileptiform activities of the different cell types should be identified. The possibility of changes in the sensitivity of these various cell types to GABA and glutamate during stage 1 and 2 should be investigated. Special attention should be devoted to dentate granule cells since they appear to play a key role in delaying the transition from "interictal" to "ictal" epileptiform activities. In particular, the possibility of a gradual increase in the contribution of NMDA receptors to the epileptiform activity of dentate granule cells during stage 1 should be investigated in light of recent data suggesting a critical role of this receptor subtype in the genesis of kindling seizures (Mody and Heinemann, 1987; Stringer and Lothman, 1988). Moreover, extraand intracellular methods should be used to identify what are the special characteristics of the temporal hippocampus underlying its peculiar epileptogenic propensity.

\section{References}

Alonso A, Llinás RR (1989) Subthreshold Na-dependent theta-like rhythmicity in stellate cells of entorhinal cortex layer II. Nature 342: 175-177.

Amaral, DG (1987) Memory: anatomical organization of candidate brain regions. In: Handbook of physiology (Mountcastle VB, Plum F, eds), pp 288-321. Bethesda, MD: American Physiological Society.

Amaral DG, Witter MP (1989) The three-dimensional organization of the hippocampal formation: a review of anatomical data. Neuroscience 31:571-591.

Andersen P (1975) Organization of hippocampal neurons and their interconnections. In: The hippocampus (Isaacson RL, Pribram KH, eds), pp 155-175. New York: Plenum.

Andersen P, Holmqvist B, Voorhoeve, PE (1966a) Entorhinal activation of dentate granule cells. Acta Physiol Scand 66:448-460.

Andersen P, Holmqvist B, Voorhoeve PE (1966b) Excitatory synapses on hippocampal apical dendrites activated by entorhinal stimulation. Acta Physiol Scand 66:461-472.

Andersen P, Bliss VP, Skrede K (1971) Lamellar organization of hippocampal excitatory pathways. Exp Brain Res 13:222-238.

Bartesaghi R, Gessi T, Sperti L (1983) Interlamellar transfer of impulses in the hippocampal formation. Exp Neurol 82:550-567.

Bragdon AC, Taylor DM, Wilson WA (1986) Potassium-induced epileptiform activity in area CA3 varies markedly along the septotemporal axis of the rat hippocampus. Brain Res 378:169-173.

Buzsáki $G$ (1986) Hippocampal sharp waves: their origin and significance. Brain Res 398:242-252.

Buzsáki G (1989) Two-stage model of memory trace formation: a role for noisy brain states. Neuroscience 31:551-570.

Buzsáki G, Leung LS, Vanderwolf CH (1983) Cellular bases of hippocampal EEG in the behaving rat. Brain Res Rev 6:139-171. 
Buzsáki G, Chen LS, Gage FH (1990) Spatial organization of physiological activity in the hippocampal region: relevance to memory formation. Prog Brain Res 83:257-268.

Buzsáki G, Hsu M, Slamka C, Gage FH, Horváth Z (1991) Emergence and propagation of interictal spikes in the subcortically denervated hippocampus. Hippocampus 1:163-180.

deCurtis M, Paré D, Llinás RR (1991) The electrophysiology of the olfactory-hippocampal circuit in the isolated and perfused adult mammalian brain in vitro. Hippocampus 1:341-354.

Delgado-Escueta AV, Walsh GO (1983) The selection process for surgery of intractable complex partial seizures: surface and depth electrography. In: Epilepsy (Penry JK, Purpura D, eds), pp 295-326. New York: Raven.

Delgado-Escueta AV, Ward AA, Woodbury DM, Porter RJ (1986) Basic mechanisms of the epilepsies. New York: Raven.

Finch DM, Babb TL (1977) Response decrement in a hippocampal basket cell. Brain Res 130:354-359.

Garcia-Austt F, Arana R, Migliaro E, Sande MT, Segundo JP (1954) Changes in the EEG and in the tendon jerks induced by the stimulation of the fornix in man. Electroencephalogr Clin Neurophysiol 6:653661.

Gilbert M, Racine RJ, Smith GK (1985) Epileptiform burst responses in ventral vs. dorsal hippocampal slices. Brain Res 361:389-391.

Horikawa K, Armstrong WE (1988) A versatile means of intracellular labeling: injection of biocytin and its detection with avidin conjugates. Neuroscience 25:1-11.

Ishizuka N, Weber J, Amaral DG (1990) Organization of intrahippocampal projections originating from $\mathrm{CA} 3$ pyramidal cells in the rat. I Comp Neurol 295:580-623.

Jones RSG, Heinemann U (1988) Synaptic and intrinsic responses of medial entorhinal cortical cells in normal and magnesium-free medium in vitro. J Neurophysiol 59:1476-1496.

Jones RSG, Lambert JDC (1989) Involvement of excitatory amino acid receptors in epileptiform activity in the rat entorhinal cortex and dentate gyrus in vitro. Br J Pharmacol 98:835P.

Jones RSG, Lambert JDC (1990) The role of excitatory amino acid receptors in the propagation of epileptiform discharges from the entorhinal cortex to the dentate gyrus in vitro. Exp Brain Res 80:310322.

Lacaille JC, Kunkel DD, Schwartzkroin PA (1989) Electrophysiological and morphological characterization of hippocampal interneurons. In: The hippocampus: new vistas, pp 287-305. New York: Liss.

Laurberg S (1979) Commissural and intrinsic connections of the rat hippocampus. J Comp Neurol 184:685-708.

Laurberg S, Sorensen KE (1981) Associational and commissural collaterals of neurons in the hippocampal formation (hilus fasciae dentatae and subfield CA3). Brain Res 212:287-300.

Lee PHK, Xie CW, Lewis DV, Wilson WA, Mitchell CL, Hong JS (1990) Opioid-induced epileptiform bursting in hippocampal slices: higher susceptibility in ventral than dorsal hippocampus. J Pharmacol Exp Ther 253:545-551.

Liberson WT, Scoville WB, Dunsmore RH (1951) Stimulation studies of the prefrontal lobe and uncus in man. Electroencephalogr Clin Neurophysiol 7:211-222.

Llinás R, Yarom Y, Sugimori M (1981) The isolated mammalian brain in vitro: a new technique for the analysis of the electrical activity of neuronal circuit function. Fcd Proc 40:2240-2245.

Llinás R, Muhlethaler M, Walton K (1989) Electrophysiology of the isolated adult guinea pig in vitro. J Physiol 414:16P.

Lorente de Nó R (1933) Studies on the structure of the cerebral cortex. I. The area entorhinalis. J Psychol Neurol 45:381-438.

Lorente de Nó R (1934) Studies on the structure of the cerebral cortex. II. Continuation of the study of the ammonic system. J Psychol Neurol 46:113-117.

Lothman EW, Collins RC (1981) Kainic acid induced limbic seizures: metabolic, behavioral, electroencephalographic and neuropathologic correlates. Brain Res 218:299-318.

MacVicar BA, Dudek FE (1982) Electrotonic coupling between granule cells of the rat dentate gyrus: physiological and anatomical evidence. J Neurophysiol 47:579-592.

Madison DV, Malcnka RC, Nicoll RA (1991) Mechanisms underlying long-term potentiation of synaptic transmission. Annu Rev Neurosci 14:379-397.

Miles R, Wong RKS (1987) Inhibitory control of local excitatory circuits in the guinea pig hippocampus. J Physiol (Lond) 338:611629.

Mody I, Heinemann U (1987) NMDA receptors of dentate gyrus granule cells participate in synaptic transmission following kindling. Nature 326:701-704.

Mody I, Lambert JDC, Heinemann U (1987) Low extracellular magnesium induces epileptiform activity and spreading depression in rat hippocampal slices. J Neurophysiol 57:869-888.

Muhlethaler M, Serafin M (1990) Thalamic spindles in an isolated and pcrfused preparation in vitro. Brain Res 524:17-21.

Paré D, Llinás RR (1991) Spatiotemporal temporal organization of the entorhinal input to the hippocampus: multiple electrode recording from the adult guinea pig brain maintained in vitro. Soc Neurosci Abstr 17:52.11.

Ramón y CajalS (1893) Estructura del asta de Ammon y fascia dentata. Ann Soc Esp Hist Nat 22.

Rutecki PA, Lebda FJ, Johnston D (1985) Epileptiform activity induced by changes in extracellular potassium in hippocampus. $J$ Neurophysiol 54:1363-1374.

Ruth RE, Collier TJ, Routtenberg A (1982) Topography between the entorhinal cortex and the dentate septotemporal axis in rats: $I$. Medial and intermediate entorhinal projecting cells. J Comp Neurol 209:6978.

Schmalbruch H, Jahnsen H (1981) Gap junctions on CA3 pyramidal cells of guinea-pig hippocampus shown by freeze fracture. Brain Res 217:175-178.

Schwartzkroin PA (1977) Further characteristics of hippocampal CA1 cells in vitro. Brain Res 128:53-68.

Schwartzkroin PA, Mathers LH (1978) Physiological and morphological identification of a nonpyramidal cell type. Brain Res 157:110.

Schwartzkroin PA, Prince DA (1978) Cellular and ficld potential properties of epileptogenic hippocampal slices. Brain Res 147:117-130.

Stanton PK, Jones RSG, Mody I, Heinemann U (1986) Epileptiform activity induced by lowering extracellular magnesium in combined hippocampal-entorhinal cortex slices: modulation by receptors for norepinephrine and $N$-methyl-D-aspartate. Epilepsy Res 1:53-62.

Stelzer A, Slater NT, ten Bruggencate G (1987) Activation of NMDA receptors blocks GABAergic inhibition in an in vitro model of epilepsy. Nature 326:698-701.

Stringer JL, Lothman EW (1988) NMDA receptor dependent paroxysmal discharges in the dentate gyrus. Neurosci Lett 92:69-75.

Stringer JL, Lothman EW (1989) Maximal dentate gyrus activation: characteristics and alterations after repeated seizures. J Neurophysiol 62:136-143.

Stringer JL, Lothman EW (1990) Maximal dentate activation: a tool to screen compounds for activity against limbic seizures. Epilepsy Res 5:169-176.

Stringer JL, Williamson JM, Lothman EW (1989) Induction of paroxysmal discharges in the dentate gyrus: frequency dependence and relationship to afterdischarge production. J Neurophysiol 62:126135 .

Stringer JL, Williamson JM, Lothman EW (1991) Maximal dentate activation is produced by amygdala stimulation in unanesthetized rats. Brain Res 542:336-342.

Suzuki SS, Smith GK (1987) Spontaneous EEG spikes in the normal hippocampus. I. Behavioral correlates, laminar profiles and bilateral synchrony. Electroencephalogr Clin Neurophysiol 67:348-359.

Swanson LW, Wyss JM, Cowan WM (1978) An autoradiographic study of the organization of the intrahippocampal association pathways in the rat. J Comp Neurol 181:681-716.

Teyler TJ, DiScenna P (1987) Long-term potentiation. Annu Rev Neurosci 10:131-161.

Thompson SM, Gahwiler BH (1989) Activity-dependent disinhibition. III. Desensitization and GABA $_{\mathrm{B}}$ receptor-mediated presynaptic inhibition in the hippocampus in vitro. J Neurophysiol 61:524-533.

Traub RD, Miles R, Wong RKS, Schulman LS, Schneiderman JH (1987) Model of synchronized hippocampal bursts in the presence of inhibition. II. Ongoing spontaneous population events. J Neurophysiol 58:752-765.

Voskuyl RA, Albus H (1985) Spontaneous epileptiform discharges in hippocampal slices induced by 4-aminopyridine. Brain Res 342:5466.

Walsh GO, Delgado-Escueta AV (1984) Type II complex partial sei- 
zures: poor results of anterior temporal lobectomy. Neurology 34:113.

Walther H, Lambert JDC, Jones RSG, Heinemann U, Hamon B (1986) Epileptiform activity in combined slices of the hippocampus, subiculum and entorhinal cortex during perfusion of low magnesium medium. Neurosci Lett 69:156-161.

Witter MP, Groenewegen HJ, Lopes da Silva FH, Lohman AHM (1989) Functional organization of the extrinsic and intrinsic circuitry of the parahippocampal region. Prog Neurobiol 33:161-253.

Wong RKS, Prince DA (1978) Participation of calcium spikes during intrinsic burst firing in hippocampal neurons. Brain Res 159:385390.
Wong RKS, Traub RD (1983) Synchronized burst discharge in the disinhibited hippocampal slice. I. Initiation in the CA2-CA3 region. J Neurophysiol 49:459-471.

Wong RKS, Watkins D (1982) Cellular factors influencing GABA response in hippocampal pyramidal cells. J Neurophysiol 48:938951.

Wyss JM (1981) An autoradiographic study of the efferent connections of the entorhinal cortex in the rat. J Comp Neurol 199:495-512.

Yoshida K (1984) Influences of bilateral hippocampal lesions upon kindled amygdaloid convulsive seizure in rats. Physiol Behav 32:123126. 